\title{
From Mindsponge Process to Discovery
}

\author{
Minh-Hoang Nguyen \\ Centre for Interdisciplinary Social Research \\ Phenikaa University \\ Yen Nghia, Ha Dong, Hanoi 100803, Vietnam

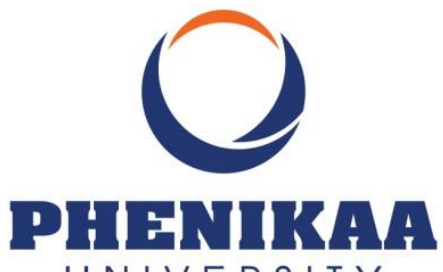 \\ UNIVERSIT Y
}

February 11, 2021

Just a few days ago, my colleagues and I completed a manuscript titled "Alice in Suicideland: Exploring the Suicidal Ideation Mechanism through the Sense of Connectedness and Help-Seeking Behaviors" [1]. In the manuscript, we explain how the suicidal ideation mechanism happens in the students' minds and how suicidal ideation can be prevented by help-related information employing the Mindsponge mechanism [2,3]. Though thought-provoking, the mechanism obtains high complexity and dynamics and might be subversive to the existing literature in Psychological sciences. But, how did I come up with such a discovery?

To develop the suicidal ideation mechanism, I had been through a long journey with many up and down. However, everything starts with an objective. The idea of using the Mindsponge mechanism for explaining mental and psychological phenomena have germinated in my mind since the first publications on mental health two years ago. When my mentor introduced me to Mindsponge, I was immediately attracted to its potential implications in explaining human psychology and behaviors. Unfortunately, due to my lack of experience and knowledge in the field as well as being busy with other projects, I could not pursue the idea eventually. Still, the question of whether the Mindsponge mechanism can explain a human's psychology and behaviors persisted in occupying a place in my mind. 
Just like the finding in my paper, the appearance of any thought is the result of information availability, filtering process, and cost-benefit judgment. To elaborate, getting to know and understand the Mindsponge mechanism, mental health problems, and how to employ Bayesian analysis is the first fundamental criterion that I need to acquire. Without the two years of absorbing the necessary knowledge, I could not even think of the question. During the information absorbing process, conflicts happened frequently, so withstanding the pressure and facing various challenges were inevitable to achieve the final goal (the idea). Just imagine that if I quitted one day or two days before the 'due date' due to difficulties, I would have never thought of the idea. Thus, a high working discipline is really essential.

During the last two years, the amount of information around me was tremendous, so filtering and emitting "endless" unnecessary information is, indeed, essential to keep myself from becoming paranoid [4]. But how can I choose which information to learn or unlearn? I think the filtering process is driven by my cost-benefit judgment based on the contextual evidence and prior experience. In particular, information about Bayesian analysis persists existing in my mind because of its advantages [5-7] and my familiarity with the package employed [8]. It is so unreasonable to use another method or package that consumes more time and effort to learn and is not our team's original package [9]. The Mindsponge mechanism is also similar. It stays in my mind because I have felt that the mechanism is a grand theory and full of potential for understanding human psychology. Moreover, I prefer the mechanism over other existing theories in the literature as it is developed by my mentor - who inspires and instructs me a lot of knowledge and wisdom. Apparently, my cost-benefit judgment is not only affected by rationality but also emotionality.

Is the idea an outcome of the information accumulation process? Not exactly, the sole information accumulation process is a vital component but not sufficient to create the idea. I think a 'special moment' is another component. I assume it to be a 'special moment' but not a type of information because luck is involved. Notably, the idea came up in my mind when Tako - my friend, and I were joking with each other about the book chapter that we were planning to write.

Interestingly, I should have written that book chapter two months before the 'special moment,' but because of many reasons, I needed to postpone it until then. Without talking to my friends at the right moment and the problems that hinder me from completing the book chapter, the idea could not appear. In a short note, my friend also reflected on the process of writing the paper [10].

To sum up, the idea of a suicidal mechanism is an outcome of the learning and unlearning process, directed by an objective, maintained by a high working discipline, and actualized by a 'special moment.' Of course, my mentor and colleagues' support is also a 
primary factor that helps me actualize the idea. Since the idea was born, many valuable ideas about studying human cognitive and psychological development have started to come up in my mind. This, perhaps, is the strategic advantage of serendipity [11].

In just a few more hours, I will celebrate the 2021 Vietnamese lunar new year. I hope this essay will be a good note after 2020, full of difficulties. By making use of the strategic advantage of coming up with a new valuable idea, I also hope to explore more significant ideas in the future.

My mind is like a light bulb. When the light is turned on, it is time to turn up the brightness.

\section{References}

[1] Minh-Hoang Nguyen et al. (2021). Alice in Suicideland: Exploring the Suicidal Ideation Mechanism through the Sense of Connectedness and Help-Seeking Behaviors. International Journal of Environmental Research and Public Health, 18(7), 3681.

[2] Quan-Hoang Vuong. (2016). Global mindset as the integration of emerging sociocultural values through mindsponge processes: A transition economy perspective. In J. Kuada (Ed.), Global Mindsets: Exploration and Perspectives (pp. 109-126). London: Routledge.

[3] Quan-Hoang Vuong \& Nancy K. Napier. (2015). Acculturation and global mindsponge: an emerging market perspective. International Journal of Intercultural Relations, 49, 354-367.

[4] Manh-Toan Ho. (2021). The paranoia of endless data. OSF Preprints. DOI: 10.31219/osf.io/tx4bw.

[5] Richard McElreath. (2020). Statistical rethinking: A Bayesian course with examples in $R$ and Stan. CRC press.

[6] Laura Uusitalo. (2007). Advantages and challenges of Bayesian networks in environmental modelling. Ecological Modelling, 203(3-4), 312-318.

[7] Eric-Jan Wagenmakers et al. (2018). Bayesian inference for psychology. Part I: Theoretical advantages and practical ramifications. Psychonomic Bulletin Review, 25(1), 35-57.

[8] Quan-Hoang Vuong, et al. (2020). Bayesian analysis for social data: A step-by-step protocol and interpretation. MethodsX, 7, 100924.

[9] Viet-Phuong La \& Quan-Hoang Vuong. (2019). bayesvl: Visually learning the graphical structure of Bayesian networks and performing MCMC with 'Stan'. The 
Comprehensive $R$ Archive Network (CRAN). Available from: https://cran.rproject.org/web/packages/bayesvl/index.html.

[10] Tam-Tri Le. (2021). I am losing (and gaining) my mind: On the philosophy of Mindsponge. OSF Preprints. DOI: 10.31219/osf.io/hu69z.

[11] Nancy K. Napier \& Quan-Hoang Vuong. (2013). Serendipity as a Strategic Advantage?. Strategic Management in the 21st Century, 1, 175-199. 